\title{
Association of amino acids with common complications of prematurity
}

\author{
Kelli K. Ryckman', John M. Dagle', Oleg A. Shchelochkov' ${ }^{1}$ Noah Ehinger², Stanley D. Poole², Stanton L. Berberich³, Jeff Reese \\ and Jeffrey C. Murray'
}

BACKGROUND: Tandem mass spectrometry has been proposed as a method of diagnosing or predicting the development of common complex neonatal diseases. Our objective was to identify metabolites associated with common complications of prematurity.

METHODS: We performed a retrospective analysis of medical data and metabolite measurements from routine neonatal screening on 689 preterm ( $<37$ wk of gestational age) neonates.

RESULTS: We observed higher levels of phenylalanine (PHE) in infants with respiratory distress syndrome (RDS; $P=1.7 \times 10^{-5}$ ), the only association that was significant after correction for multiple testing. We found suggestive significance $(P<0.001)$ of higher essential amino acids in infants with patent ductus arteriosus (PDA). Functionality of these findings was explored in the ductus arteriosus (DA) isolated from term and preterm mouse pups. None of the amino acids had a direct vasodilatory effect on the isolated DA.

CONCLUSION: We found that newborns with RDS had higher levels of PHE that may be a result of impaired PHE hydroxylase activity. We also detected marginally higher levels of all measured essential amino acids in infants with PDA. We did not find dilation of the mouse ductus for these metabolites, indicating that instead of potentially causing PDA, they are probably serving as markers of catabolism.

C omprehensive metabolic profiling at birth is a critical public health program nationally, as well as internationally, to detect rare congenital conditions that, if identified early, can be treated. If left untreated, these disorders can cause lifelong morbidities or death. In addition to state-mandated newborn screening, longitudinal metabolic profiling with high-throughput methods such as tandem mass spectrometry may prove critical for monitoring, diagnosing, and treating conditions as they develop in the neonatal intensive care unit (1-3). Although several metabolites are currently used for routine monitoring of overall health in the neonatal intensive care unit, including glucose, blood gas values, and electrolytes, the measurement of analytes with high-throughput methods used in newborn screening programs, such as tandem mass spectrometry, has not yet been implemented for monitoring preterm infant health or potential risk for common complex diseases.

Preterm and/or sick neonates are known to have distinct metabolic profiles, often defined by amino acid and acylcarnitine measurements, as compared with their term and/ or healthy counterparts $(4,5)$. Although stress and immature liver and kidney function may explain some of the observed metabolic differences, there are probably many other factors contributing to an infant's metabolism at birth, including fetal and maternal influences, perinatal events, and genetic background. Gestational age and birth weight are known contributors to variation in metabolic profiles; however, few studies have examined specific conditions that often accompany low birth weight and early gestational age (3,6-9). A few studies have applied nuclear magnetic resonance spectroscopy analysis of urine to examine neonates with patent ductus arteriosus (PDA), intrauterine growth restriction, or asphyxiation, and children with neuropathies $(3,6-9)$. This technique offers a promising approach for distinguishing patterns of metabolites altered in specific neonatal diseases and conditions.

State-wide neonatal screening is generally performed from dried blood spots collected 1-3 d after birth. Many metabolites are identified through expanded newborn screening using tandem mass spectrometry. To our knowledge, no study has used these values to examine associations with complications commonly associated with prematurity. Our objective was to determine whether metabolites from routine newborn screening are associated with common diseases of prematurity and could therefore be potential biomarkers or therapeutic targets for critical illnesses in the neonatal intensive care unit. We examined metabolite measurements from routine newborn screening in 689 preterm $(<37$ wk gestation) newborns to identify metabolite associations with common complications of prematurity, including PDA, respiratory distress syndrome (RDS), and necrotizing enterocolitis. We followed up metabolic findings with PDA using a previously established mouse model to test ductus arteriosus (DA) contractility. There are several different knockout mouse models that have PDA; each has respiratory distress, pulmonary congestion, and possible fatality if a 
Table 1. List of analytes examined

\begin{tabular}{|c|c|}
\hline Amino acids ( $\mu \mathrm{mol} / \mathrm{l})$ & Acylcarnitines $(\mu \mathrm{mol} / \mathrm{l})$ \\
\hline Alanine & Hexanoylcarnitine \\
\hline Arginine & Methylglutarylcarnitine \\
\hline Argininosuccinate $^{\mathrm{a}}$ & Octanoylcarnitine \\
\hline Citrulline & Octenoylcarnitine \\
\hline Glutamate & Suberylcarnitine ${ }^{b}$ \\
\hline Glycine $^{b}$ & Decanoylcarnitine \\
\hline Leucine & Decenoylcarnitine \\
\hline Methionine & Decadienoylcarnitine $^{\mathrm{b}}$ \\
\hline Ornithine $^{b}$ & Dihydrosebacylcarnitine $^{\mathrm{b}}$ \\
\hline 5-Oxoproline ${ }^{b}$ & Dodecanoylcarnitine \\
\hline Phenylalanine & Dodecenoylcarnitine \\
\hline Tyrosine & Tetradecanoylcarnitine \\
\hline Valine & Tetradecenoylcarnitine \\
\hline Acylcarnitines $(\mu \mathrm{mol} / \mathrm{l})$ & Tetradecadienoylcarnitine \\
\hline Free carnitine & 3-Hydroxytetradecanoylcarnitine ${ }^{a}$ \\
\hline Acetylcarnitine & Palmitoylcarnitine \\
\hline Propionylcarnitine & Palmitoleylcarnitine \\
\hline Malonylcarnitine & 3-Hydroxypalmitoleylcarnitine ${ }^{a}$ \\
\hline $\begin{array}{l}\text { Butyrylcarnitine + } \\
\text { isobutyrylcarnitine }\end{array}$ & 3-Hydroxypalmitoylcarnitine ${ }^{a}$ \\
\hline Methylmalonylcarnitine & Stearoylcarnitine \\
\hline 3-Hydroxybutyrylcarnitine ${ }^{b}$ & Oleoylcarnitine \\
\hline $\begin{array}{l}\text { Isovalerylcarnitine + } \\
\text { methylbutyrylcarnitine }\end{array}$ & 3-Hydroxyoleoylcarnitine ${ }^{a}$ \\
\hline Tiglylcarnitine $^{\mathrm{a}}$ & Linoleoylcarnitine \\
\hline Glutarylcarnitine & 3-Hydroxylinoleoylcarnitine ${ }^{b}$ \\
\hline 3-Hydroxyisovalerylcarnitine & 3-Hydroxystearoylcarnitine ${ }^{a}$ \\
\hline
\end{tabular}

aResults are not presented for these metabolites that had low variability (SD $\leq 0.01$ $\mu \mathrm{mol} / \mathrm{l})$. ${ }^{b}$ Metabolites added or dropped from the tandem mass spectrometry panel within the collection period.

large PDA cannot be treated or rescued. We tested the vasodilatory effects of candidate metabolites on the mouse DA to estimate the functionality of our metabolic associations.

\section{RESULTS}

The metabolites examined are presented in Table 1, and demographic characteristics of the cohort are described in Table 2. Associations with demographic and clinical factors were examined for each metabolite after adjustment for gestational age, birth weight, year of sample collection, and change in the assay during the study period. Increased concentrations of free carnitine $(\mathrm{C} 0)$ and multiple acylcarnitines (acetylcarnitine (C2), methylmalonylcarnitine (C4-DC), tetradecanoylcarnitine (C14), and lindeoylcarnitine (C18:2)) were observed in males as compared with females (Table 3). Several other acylcarnitines were either higher (methylglutarylcarnitine (C6-DC): $P=8.5 \times 10^{-7}$, dodecanoylcarnitine (C12): $P=6.2 \times 10^{-8}$, and 3-hydroxyleoylcarnitine $\left.(\mathrm{C} 18: 2-\mathrm{OH}): P=2.0 \times 10^{-9}\right)$ or lower (C4-DC: $P=1.5 \times 10^{-5}$ ) in births taking place in the summer
Table 2. Demographic characteristics

\begin{tabular}{|c|c|c|c|}
\hline Variable & $\begin{array}{l}\text { Mean } \pm \text { SD } \\
\text { or } n(\%)\end{array}$ & $\begin{array}{c}\text { Number of } \\
\text { observations }\end{array}$ & $\%$ Missing \\
\hline Gestational age (wk) & $31.3 \pm 3.3$ & 688 & 0.1 \\
\hline Birth weight (g) & $1,767 \pm 719$ & 689 & 0.0 \\
\hline Infant gender (male) & $380(55.2 \%)$ & 689 & 0.0 \\
\hline Infant race (Caucasian) & $592(87.8)$ & 674 & 2.2 \\
\hline Twin/multiple & 130 (18.9\%) & 689 & 0.0 \\
\hline Season of birth & & 689 & 0.0 \\
\hline $\begin{array}{l}\text { Winter (December to } \\
\text { February) }\end{array}$ & $197(28.6 \%)$ & & \\
\hline Spring (March to May) & 141 (20.5\%) & & \\
\hline $\begin{array}{l}\text { Summer (June to } \\
\text { August) }\end{array}$ & $164(23.8 \%)$ & & \\
\hline $\begin{array}{l}\text { Fall (September to } \\
\text { November) }\end{array}$ & $187(27.1 \%)$ & & \\
\hline $\begin{array}{l}\text { Age }(h) \text { at the time of } \\
\text { sample collection }\end{array}$ & $29.3 \pm 6.9$ & 689 & 0.0 \\
\hline APGAR score at $1 \mathrm{~min}$ & $6.2 \pm 2.1$ & 681 & 1.2 \\
\hline APGAR score at $5 \mathrm{~min}$ & $7.9 \pm 1.3$ & 682 & 1.0 \\
\hline $\begin{array}{l}\text { Highest total bilirubin } \\
(\mathrm{mg} / \mathrm{dl})\end{array}$ & $9.5 \pm 3.1$ & 646 & 6.2 \\
\hline $\begin{array}{l}\text { Respiratory distress } \\
\text { syndrome }\end{array}$ & $364(60.6 \%)$ & 601 & 12.8 \\
\hline Patent ductus arteriosus & $133(19.9 \%)$ & 669 & 2.9 \\
\hline Necrotizing enterocolitis & $12(2.0 \%)$ & 611 & 11.3 \\
\hline Congenital anomaly & $50(7.3 \%)$ & 689 & 0.0 \\
\hline
\end{tabular}

The number of observations represents the total number of individuals with nonmissing data for a given variable.

Table 3. Metabolite associations with gender

\begin{tabular}{lccc}
\hline & $\begin{array}{c}\text { Female } \\
(n=309)\end{array}$ & $\begin{array}{c}\text { Male } \\
(n=380)\end{array}$ & $P$ value \\
\hline Free carnitine & $22.7 \pm 9.8$ & $26.5 \pm 10.6$ & $3.2 \times 10^{-10}$ \\
Acetylcarnitine & $24.3 \pm 10.7$ & $28.3 \pm 11.5$ & $1.4 \times 10^{-8}$ \\
Methylmalonylcarnitine & $0.10 \pm 0.05$ & $0.12 \pm 0.06$ & $6.4 \times 10^{-6}$ \\
Tetradecanoylcarnitine & $0.21 \pm 0.08$ & $0.24 \pm 0.09$ & $1.6 \times 10^{-6}$ \\
Linoleoylcarnitine & $0.23 \pm 0.17$ & $0.26 \pm 0.17$ & $3.5 \times 10^{-5}$ \\
\hline
\end{tabular}

Means and SD in $\mu \mathrm{mol} / \mathrm{l}$ units. ANOVA model adjusted for major assay change, year of sample collection, and adjustment for birth weight and gestational age.

months (Figure 1). In addition, 3-hydroxyisovalerylcarnitine $(\mathrm{C} 5-\mathrm{OH})\left(P=3.2 \times 10^{-7}\right)$ was lower in twin as compared with singleton births, and C18:2 $\left(P=2.4 \times 10^{-5}\right)$ increased with increasing age at time of sample collection (Figure 1).

Figure 2 illustrates metabolite associations with complications of prematurity including RDS, PDA, and necrotizing enterocolitis. Phenylalanine (PHE) was significantly $(P=$ $1.7 \times 10^{-5}$ ) increased in infants with RDS as compared with controls, and this was the only complication of prematurity to have an association that remained significant after correction for multiple testing (Table 4). Additional adjustment for prenatal steroids did not alter the significance of this result $(P=$ 


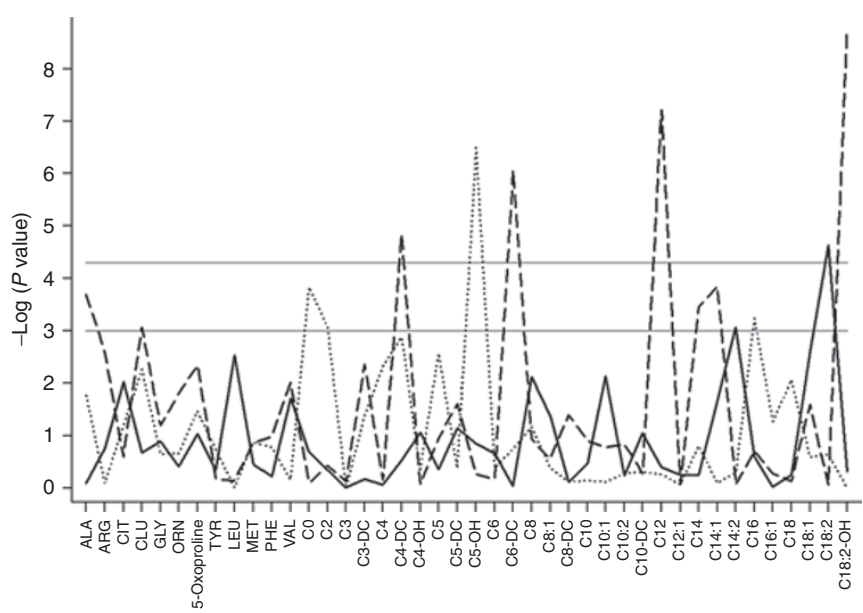

Figure 1. Metabolite associations with infant demographics. Associations are represented by the negative logarithm of the $P$ value, with the solid line representing age at time of sample collection, the dashed line representing season of birth, and the dotted line representing twin or multiple gestations. The first horizontal gray solid line, at $-\log (P)=3$, represents a $P$ value of $\leq 0.001$, and the second horizontal gray solid line, at $-\log (P)=4.3$, represents a Bonferroni corrected significance threshold of $P \leq 5 \times 10^{-5}$.

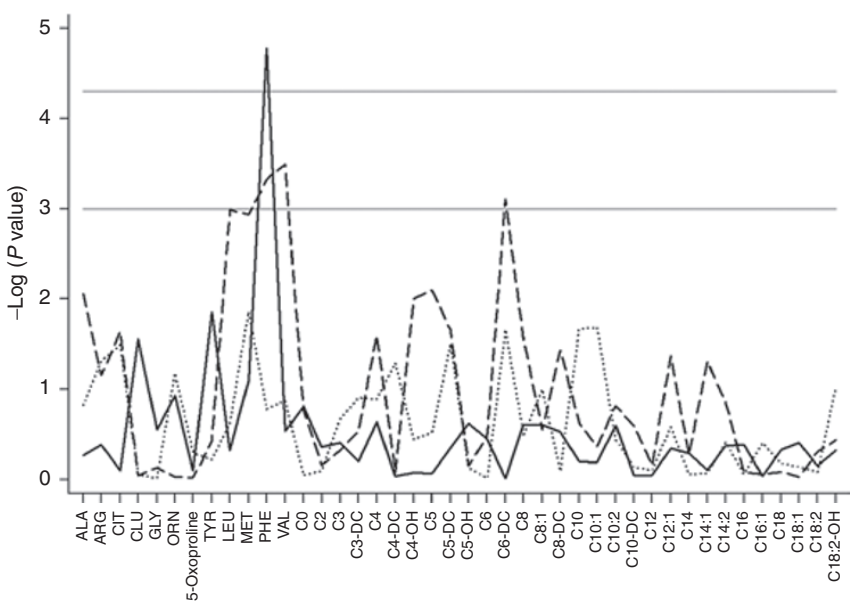

Figure 2. Metabolite associations with complications of prematurity. Associations are represented by the negative logarithm of the $P$ value, with the solid line representing respiratory distress syndrome, the dashed line representing patent ductus arteriosus, and the dotted line representing necrotizing enterocolitis. The first horizontal gray solid line, at -log $(P)=3$, represents a $P$ value of $\leq 0.001$, and the second horizontal gray solid line, at $-\log (P)=4.3$, represents a Bonferroni corrected significance threshold of $P \leq 5 \times 10^{-5}$.

Table 4. Metabolite associations with complications of prematurity

\begin{tabular}{|c|c|c|c|c|c|}
\hline RDS & No $(N=237)$ & Yes $(N=364)$ & Model $1, P$ value & Model $2, P$ value ${ }^{b}$ & Model $3, P$ value \\
\hline Phenylalanine & $61.6 \pm 18.7$ & $72.6 \pm 29.9$ & $1.7 \times 10^{-5}$ & $4.1 \times 10^{-4}$ & 0.03 \\
\hline PDA & No $(N=536)$ & Yes $(N=133)$ & & & \\
\hline Methionine & $25.8 \pm 20.5$ & $33.1 \pm 17.9$ & $1.2 \times 10^{-3}$ & 0.02 & 0.02 \\
\hline Phenylalanine & $66.7 \pm 30.4$ & $77.0 \pm 20.8$ & $4.7 \times 10^{-4}$ & $1.2 \times 10^{-3}$ & $9.3 \times 10^{-4}$ \\
\hline Valine & $93.6 \pm 53.7$ & $123.2 \pm 48.8$ & $3.2 \times 10^{-4}$ & $1.0 \times 10^{-3}$ & $8.3 \times 10^{-3}$ \\
\hline
\end{tabular}

Means and SD in $\mu \mathrm{mol} / \mathrm{l}$ units.

PDA, patent ductus arteriosus; RDS, respiratory distress syndrome; TPN, total parenteral nutrition.

aANOVA model adjusted for major assay change, year of sample collection, and adjustment for birth weight and gestational age. ${ }^{\mathrm{b}} \mathrm{ANOVA}$ model adjusted for major assay change, year of sample collection, and adjustment for birth weight and gestational age and excluding infants on TPN, twins, and infants with congenital anomalies. ' ${ }^{\mathrm{ANOV}} \mathrm{A}$ model adjusted for major assay change, year of sample collection, birth weight, and gestational age in infants $\leq 32$ wk gestation.

$\left.2.1 \times 10^{-5}\right)$. This association remained when examining infants born at $<32$ wk gestation $(P=0.03)$.

Although not significant after correction for multiple testing, it is notable that multiple essential amino acids were of suggestive significance $\left(P \leq 1.0 \times 10^{-3}\right)$. Specifically, leucine (LEU), methionine (MET), PHE, and valine (VAL) were higher in infants with PDA (Figure 2 and Table 4 ) as compared with those without. C6-DC was higher in infants with PDA $\left(P=7.8 \times 10^{-4}\right)$ and in infants born in summer. Including both covariates in the same model with C6-DC (and adjusting for birth weight, gestational age, year of collection, and change in the assay during the study period) did not decrease the significance of the association between C6-DC and PDA $\left(P=5.6 \times 10^{-3}\right)$. Although $15.0 \%$ of the infants with PDA received total parenteral nutrition before the newborn screen, excluding these individuals $(n=67)$ from the analysis did not alter the significance of the results (Table 4). Results also remained significant when examining only infants born at $<32 \mathrm{wk}$ gestation. We further followed associations with essential amino acids and PDA with functional studies in the mouse. The vascular response to L-VAL, L-MET, L-PHE, and L-LEU was examined in the isolated term or preterm DA. Exposure to cumulative doses of each compound failed to produce a significant change in ductal tone (Figure 3).

\section{DISCUSSION}

Recently, measuring the metabolic profile in urine has been proposed as a method for monitoring progression of diseases that often develop in newborns born preterm (4). We identified specific complications of prematurity associated with essential amino acid levels. Specifically, newborns with RDS had higher levels of PHE as compared with controls. Higher PHE concentrations have also been observed in adult patients with respiratory distress $(10,11)$. PHE could also be increased in response to impaired PHE hydroxylase activity. It has been hypothesized that oxidative stress as a result of inflammation can deplete 5,6,7,8-tetrahydrobiopterin, a cofactor for this enzyme (12). 


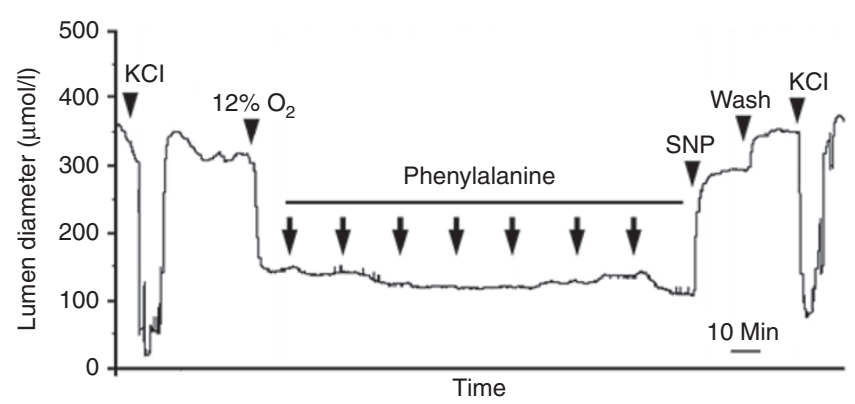

Figure 3. Response of the isolated murine ductus arteriosus to phenylalanine. Representative tracing of a term gestation mouse ductus that was mounted in a pressure myography chamber under deoxygenated conditions. Submaximal constriction of the ductus lumen diameter by oxygen was not overcome by exposure to increasing concentrations of phenylalanine $\left(10^{-9}\right.$ to $\left.10^{-3} \mathrm{~mol} / \mathrm{l}\right)$. By contrast, exposure to the nitric oxide donor sodium nitroprusside $\left(10^{-5} \mathrm{~mol} / \mathrm{l}\right)$ produced marked dilation and return to resting baseline dimensions. Terminal exposure to $50 \mathrm{mmol} / \mathrm{l}$ $\mathrm{KCl}$ confirmed responsiveness of the preparation at the completion of the study. Vertical lines represent 10-min intervals. Arrowheads indicate serial 10 -fold increases in phenylalanine concentration in the perfusion bath.

Although not significant after correction for multiple testing, we detected marginally higher levels of all measured essential amino acids (total LEU, MET, PHE, and VAL) in infants with PDA. Branched-chain amino acids, which include LEU, isoleucine, and VAL, are generated through catabolism and extrahepatic tissues, including cardiac muscle (13). We did not find dilation of the mouse ductus for these metabolites, indicating that instead of potentially causing PDA, they are probably serving as markers of catabolism, which is heightened in neonates in whom the patent ductus is failing to close. This result is intriguing because recent reports have suggested that persistent patency of the DA at 3-4 d of life can be predicted using the first urine passed by preterm neonates (9). Although the study does not reveal the specific metabolites associated with the prediction, it asserts not only that using a metabolomics approach can predict PDA but also that it can distinguish the responders from the nonresponders to ibuprofen therapy (9). Because our dried blood spot samples are taken at 1-3 d of life, right before the identification of persistent patency of the ductus, we may be detecting the distinguishing metabolic patterns that are also observed in urine samples.

A neonate's metabolism continues to evolve after birth and is influenced by a host of factors, including maternal metabolism, nutrition, toxicological insults, perinatal events, and genetic background. Consistent with previous reports, we identified strong associations between the majority of measured metabolites and gestational age and birth weight $(4,5,14)$. Specifically, amino acids increased and medium- and long-chain acylcarnitines decreased with decreasing gestational age and birth weight. Likely explanations for these differences are immature liver, kidney, and adrenal function. In addition, we found significantly lower acylcarnitine levels in females as compared with males. Several studies have shown that in adults, total carnitine is lower in females as compared with males (15-17). A possible explanation relates to estradiol levels, which have been shown to have an inverse relationship with plasma carnitine concentrations in rats (18). Our associations with the complications of prematurity were independent of potential confounders, including gestational age, birth weight, and gender.

Our study was retrospective in nature, and therefore we are not able to adequately capture or account for all potential confounding influences. In addition, we received only a snapshot of a selected group of metabolites at one time point after birth. To better assess the metabolites that we have identified as potential biomarkers for complications of prematurity, we would need longitudinal samples collected throughout the course of the condition. This was a retrospective examination of data, and several of the variables included in our analysis had a large amount of missing data points; therefore, those results would need to be interpreted with caution. One limitation of our experimental model is that the mouse DA closes more rapidly after birth than the human DA; however, in relative terms, the closure rate is similar. In addition, although prematurely born mice cannot survive outside the womb, studies on the preterm fetal DA can be performed in vitro, to determine the response to vasoactive stimuli. Although human DA tissues would be ideal, they have limited availability and viability; therefore, the mouse model of DA can serve as a surrogate for the functional assessment of human data. Our data show that neonatal essential amino acids, as measured through routine newborn screening, are elevated in certain complications that accompany preterm birth, including RDS and PDA. These metabolites are potential biomarkers for the presence and severity of several complications of prematurity.

\section{METHODS}

\section{Study Population}

This is a retrospective analysis of data collected between 2001 and 2009 as part of a prospective cohort for studying the epidemiology and genetics of preterm birth $(19,20)$. Study samples were collected at the University of Iowa Children's Hospital in Iowa City after approval by the University of Iowa Institutional Review Board (IRB200506792). Signed informed consent was obtained from all families for enrollment. Gestational age and birth weight were obtained from the medical record. A total of 689 preterm infants were included for analysis. Infants were included in data analysis if they were born preterm (delivery at $23 \mathrm{wk}$ and $0 \mathrm{~d}$ through $36 \mathrm{wk}$ and $6 \mathrm{~d}$ gestation), had not received a blood transfusion at the time of sample collection, and had their sample collected between 24 and $72 \mathrm{~h}$ after birth. Existing data, collected by medical chart review, was evaluated for 22 medical and demographic factors; completeness of the data varied across subjects. Although exploratory analysis was performed for all examined variables and correction for multiple testing accounts for all tests performed, we do not present data on the following variables because they did not show relevant associations and had a large percentage of missing data (shown in parentheses): length at birth (30.9\%), occipital frontal circumference $(29.0 \%)$, feeding method at time of newborn screen (20.8\%), highest direct bilirubin (70.4\%), hemoglobin at birth (52.1\%), bronchopulmonary dysplasia (30.6\%), intraventricular hemorrhage (37.9\%), periventricular leukomalacia (21.6\%), retinopathy of prematurity $(30.2 \%)$, and sepsis $(62.0 \%)$.

\section{Diagnosis of Complications of Prematurity}

All infants born at $<28$ wk gestational age were examined by a pediatric cardiologist using echocardiography between days 5 and 7 of life, regardless of symptoms. Infants born at $\geq 28$ weeks gestation were examined by echocardiography between days 5 and 7 of life if 
a murmur suggestive of a PDA was present. Any preterm infant was examined using echocardiography if he or she had symptoms of congestive heart failure. PDA was diagnosed if flow was detected through the DA. No infant received prophylactic indomethacin (i.e., in the first $2 \mathrm{~d}$ of life). RDS was defined radiologically in combination with the requirement of supplemental oxygen for $2 \mathrm{~h}$ or more. Surfactant was not administered as prophylaxis, but if treatment was needed, it occurred within the first hour after birth. Necrotizing enterocolitis was described using the modified Bell staging criteria (21). A stage of $2 \mathrm{a}$ or greater was considered positive for necrotizing enterocolitis.

\section{Tandem Mass Spectrometry Data}

Data on analyte measurements were provided by the State of Iowa Hygienic Laboratory and linked to the clinical medical record data. Approval for use of the data provided by the State of Iowa Hygienic Laboratory was granted by the Iowa Department of Public Health. Newborn dried blood spot specimens are collected, dried, and handled according to the Clinical Laboratory Standards Institute guideline (22). Screening procedures in Iowa are based on previously established methodology (23-25). Briefly, a derivatization method is used in which butyl esters of acylcarnitines and amino acids are prepared from the extracts. Tandem mass spectrometry is performed with Waters Quattro Micro triple quadrupole tandem mass spectrometers (Milford, MA) equipped with an electrospray ionization source operated in the positive-ion mode. Multiple reaction-monitoring mode is used to scan for specific mass ion intensities. Concentrations are obtained from the ratio of ion intensity (counts per second) at the mass that represents a specific analyte as compared with its isotopically labeled internal standard and correcting for blood volume in a $1 / 8$-inch dried blood spot punch. Both internal and external spiked control specimens, a normal control specimen, and a blank are analyzed with each batch of specimens. The external spiked control specimens are obtained from the Newborn Screening Quality Assurance Program at the US Centers for Disease Control and Prevention. Data were provided on 13 amino acids and 36 acylcarnitines (Table 1).

\section{Statistical Analysis}

Statistical modeling techniques were applied to identify maternal factors and conditions that may significantly influence neonatal metabolic profiles. All metabolites were transformed with the Box-Cox transformation to normalize each distribution. Multiple statistical modeling methods were compared, and few differences were identified; therefore, ANOVA was performed adjusting for year of sample collection, assay lot changes, gestational age, and birth weight. The significance of our results was not greatly affected when including only gestational age, only birth weight, or both covariates in the model. Standardized residuals were examined for outliers, and measurements that were $<-3.5$ or $>3.5$ SDs from the mean were removed. Analysis included 924 ANOVA models ( 22 clinical variables $\times 42$ analyte and ratio measurements), and a Bonferonni significance threshold of $P<5 \times 10^{-5}$ was used to correct for multiple testing. All analyses were performed with Stata, version 12.0 (Stata, College Station, TX). Eighteen infants were identified as having an abnormal tandem mass spectrometry test result on the first screen after birth, of whom 17 had a normal repeat screen and thus were interpreted as false-positive results; one infant with a positive screen was diagnosed with mediumchain acylcarnitine-CoA dehydrogenase deficiency. None of the significantly associated factors increased the false-positive rate for the expanded newborn screening panels.

\section{Functional Studies in the Mouse}

Adult female CD1 mice (Charles River, Raleigh, NC) were bred to produce timed pregnancies. Dams were anesthetized by intraperitoneal injection of $0.4 \mathrm{ml}$ of $2.5 \%$ avertin (2,2,2-tribromoethanol in tert-amyl alcohol; Sigma-Aldrich, St. Louis, MO), followed by isoflurane inhalation (Baxter, Deerfield, IL) to facilitate fetal anesthesia. Pregnant dams were euthanized by cervical dislocation, and offspring were delivered by uterine incision. Experiments were conducted in accordance with US National Institutes of Health animal care standards and were approved by the institutional animal care and use committee at Vanderbilt University. L-VAL, L-MET, L-PHE, and LLEU (Sigma-Aldrich) were prepared as concentrated stock solutions in aqueous buffer. Final solvent concentration was limited to $0.01 \%$ and was not expected to alter ex vivo study results (26).

Response of the isolated DA was examined by pressurized vessel myography, as previously described (26). Briefly, anesthetized fetal mouse pups were delivered at term (day 19) and preterm (day 17) gestation by cesarean section and immediately placed in ice-cold modified Krebs buffer that had been gassed with a $5 \% \mathrm{CO}_{2} / 95 \%$ nitrogen mix (deoxy Krebs) to maintain stable $\mathrm{pH}$ and mimic in utero $\mathrm{O}_{2}$ conditions. The DA, along with a portion of the transverse aorta, and the pulmonary arteries were then dissected free while submerged in cold deoxy Krebs, to maintain ductus relaxation. The ductus was then mounted on glass cannulae $(150-170 \mu \mathrm{m})$ in a custom-made chamber designed for pressure myography (Instrumentation and Model Facility, University of Vermont, Burlington, VT) and secured with single-stranded nylon sutures derived from a larger braid. The chamber was then placed on an inverted microscope where continuous measurements of the internal diameter and distending pressure were made $(100 \times)$ (IonOptix, Milton, MA). Pressure was applied to the proximal inlet of the secured vessel using an elevated column of Krebs buffer and measured using a calibrated manometer (Living Systems Instrumentation, Burlington, VT). The ductus was superfused $(6 \mathrm{ml} / \mathrm{min})$ with Krebs solution at $36.5-37.5^{\circ} \mathrm{C}$. After an equilibration period of $\sim 40 \mathrm{~min}$ at $37{ }^{\circ} \mathrm{C}$ and $5 \mathrm{~mm} \mathrm{Hg}$ of pressure, a stepwise elevation of pressure was applied, up to a working pressure of $20 \mathrm{~mm} \mathrm{Hg}$. The isolated ductus was subsequently challenged with two consecutive doses of $50 \mathrm{mmol} / \mathrm{l} \mathrm{KCl}$ to verify contractility. Poorly responsive vessels were discarded.

Drug studies, testing 4-5 vessels per compound, were conducted in a continuously recycling circuit with a total volume of $20 \mathrm{ml}$. After equilibration, the Krebs buffer was aerated with $12 \% \mathrm{O}_{2}$ to mimic newborn oxygen conditions, which resulted in submaximal constriction of the vessel (preconstriction). Activity of the DA after each dose was monitored for $20 \mathrm{~min}$ or until stable. After the complete series of cumulative doses $\left(10^{-9}\right.$ to $\left.10^{-3} \mathrm{~mol} / \mathrm{l}\right)$, the vessel was exposed to the nitric oxide donor sodium nitroprusside $\left(10^{-5} \mathrm{~mol} / \mathrm{l}\right)$ to document the capacity for vasodilation. Subsequently, exposure to $50 \mathrm{mmol} / \mathrm{l} \mathrm{KCl}$ was used to determine viability of the preparation at the end of the experiment. Ductus preparations were responsive to vasoconstrictive stimuli, including $\mathrm{KCl}$ and increased oxygen tension. Exposure to the nitric oxide donor sodium nitroprusside at the completion of each dose-response experiment overcame oxygen-induced preconstriction and produced relaxation to baseline levels of resting tone in all experiments. Vessels returned to their original diameter after return to the start-up deoxygenated conditions. The strong responses to $\mathrm{KCl}$, oxygen, and sodium nitroprusside suggest that a significant contractile or vasodilatory effect of the study compounds would be detected using this approach.

\section{ACKNOWLEDGMENTS}

We express our gratitude to the coordinating medical and research staff at the University of lowa Hospitals and Clinics in lowa City, lowa, including a special thanks to research coordinators Laura Knosp and Susan Berends. We thank the Congenital and Inherited Disorders Advisory Committee, particularly Kim Piper for her enthusiastic support and management. We thank Sara Copeland at the Health Resources Services Administration for her guidance and support on this project. We thank Franklin Delin and Dariush Shirazi from the State Hygienic Laboratory for their assistance in the acquisition of the newborn screening data. We also thank Susie McConnell, Nancy Davin, and Erin Brothers-Smith for administrative support.

The content is solely the responsibility of the authors and does not necessarily represent the official views of the Eunice Kennedy Shriver National Institute of Child Health and Human Development or the National Institutes of Health.

\section{STATEMENT OF FINANCIAL SUPPORT}

This work was supported by grants from the March of Dimes (1-FY05-126 and 6-FY08-260), National Institutes of Health (K99 HD-065786, R01 HD52953, R01 HD-57192, and R01 HL-109199), and the Children's Miracle Network through the University of lowa (grant 2224). 


\section{REFERENCES}

1. Atzori L, Antonucci R, Barberini L, Griffin JL, Fanos V. Metabolomics: a new tool for the neonatologist. J Matern Fetal Neonatal Med 2009;22:Suppl 3: 50-3.

2. Antonucci R, Atzori L, Barberini L, Fanos V. Metabolomics: the "new clinical chemistry" for personalized neonatal medicine. Minerva Pediatr 2010;62:3 Suppl 1:145-8.

3. Fanos V, Barberini L, Antonucci R, Atzori L. Metabolomics in neonatology and pediatrics. Clin Biochem 2011;44:452-4.

4. Atzori L, Antonucci R, Barberini L, et al. 1H NMR-based metabolomic analysis of urine from preterm and term neonates. Front Biosci (Elite Ed) 2011;3:1005-12.

5. Oladipo OO, Weindel AL, Saunders AN, Dietzen DJ. Impact of premature birth and critical illness on neonatal range of plasma amino acid concentrations determined by LC-MS/MS. Mol Genet Metab 2011;104:476-9.

6. Chu CY, Xiao X, Zhou XG, et al. Metabolomic and bioinformatic analyses in asphyxiated neonates. Clin Biochem 2006;39:203-9.

7. Favretto D, Cosmi E, Ragazzi E, et al. Cord blood metabolomic profiling in intrauterine growth restriction. Anal Bioanal Chem 2012;402:1109-21.

8. Dessì A, Atzori L, Noto A, et al. Metabolomics in newborns with intrauterine growth retardation (IUGR): urine reveals markers of metabolic syndrome. J Matern Fetal Neonatal Med 2011;24:Suppl 2:35-9.

9. Fanos V, Antonucci R, Barberini L, Noto A, Atzori L. Clinical application of metabolomics in neonatology. J Matern Fetal Neonatal Med 2012;25:Suppl 1:104-9.

10. Siegel JH. Cardiorespiratory manifestations of metabolic failure in sepsis and the multiple organ failure syndrome. Surg Clin North Am 1983;63:379-99.

11. Kuo CD, Wu WG, Wang JH, Chen SM, Chiang BN. Proton nuclear magnetic resonance studies of plasma to determine metabolic status of patients with adult respiratory distress syndrome. Clin Chem 1989;35:667-70.

12. Ploder M, Neurauter G, Spittler A, Schroecksnadel K, Roth E, Fuchs D. Serum phenylalanine in patients post trauma and with sepsis correlate to neopterin concentrations. Amino Acids 2008;35:303-7.

13. Harper AE, Miller RH, Block KP. Branched-chain amino acid metabolism. Annu Rev Nutr 1984;4:409-54.

14. Illsinger S, Schmidt KH, Lücke T, Vaske B, Bohnhorst B, Das AM. Plasma and urine amino acid pattern in preterm infants on enteral nutrition: impact of gestational age. Amino Acids 2010;38:959-72.
15. Borum PR. Plasma carnitine compartment and red blood cell carnitine compartment of healthy adults. Am J Clin Nutr 1987;46: 437-41.

16. Harper P, Wadström C, Cederblad G. Carnitine measurements in liver, muscle tissue, and blood in normal subjects. Clin Chem 1993;39: 592-9.

17. Reuter SE, Evans AM, Chace DH, Fornasini G. Determination of the reference range of endogenous plasma carnitines in healthy adults. Ann Clin Biochem 2008;45(Pt 6):585-92.

18. Borum PR. Regulation of the carnitine deficiency syndromes. In: Frenkel RA, McGarry JD, eds. Carnitine Biosynthesis, Metabolism and Functions. New York: Academic Press, 1980:115-26.

19. Steffen KM, Cooper ME, Shi M, et al. Maternal and fetal variation in genes of cholesterol metabolism is associated with preterm delivery. J Perinatol 2007;27:672-80

20. Ehn NL, Cooper ME, Orr K, et al. Evaluation of fetal and maternal genetic variation in the progesterone receptor gene for contributions to preterm birth. Pediatr Res 2007;62:630-5.

21. Walsh MC, Kliegman RM. Necrotizing enterocolitis: treatment based on staging criteria. Pediatr Clin North Am 1986;33:179-201.

22. Clinical and Laboratory Standards Institute. Newborn screening for preterm, low birth weight, and sick newborns; approved guideline. CLSI Document I/LA31-A 2004;29:1-29. Wayne, PA: CLSI.

23. Turgeon C, Magera MJ, Allard P, et al. Combined newborn screening for succinylacetone, amino acids, and acylcarnitines in dried blood spots. Clin Chem 2008;54:657-64

24. Chace DH, Lim T, Hansen CR, De Jesus VR, Hannon WH. Improved MS/ MS analysis of succinylacetone extracted from dried blood spots when combined with amino acids and acylcarnitine butyl esters. Clin Chim Acta 2009;407:6-9.

25. Chace DH, DiPerna JC, Mitchell BL, Sgroi B, Hofman LF, Naylor EW. Electrospray tandem mass spectrometry for analysis of acylcarnitines in dried postmortem blood specimens collected at autopsy from infants with unexplained cause of death. Clin Chem 2001;47:1166-82.

26. Reese J, Waleh N, Poole SD, Brown N, Roman C, Clyman RI. Chronic in utero cyclooxygenase inhibition alters PGE2-regulated ductus arteriosus contractile pathways and prevents postnatal closure. Pediatr Res 2009;66:155-61. 